\title{
La Cervicosuspensión Endoscópica en el Tratamiento de la Incontinencia Urinaria de Esfuerzo
}

\author{
(INFORME PRELIMINAR) $1984-1985$
}

\section{Dres.: Antonio Lomanto Morán* y Jacinto Sánchez Angarita**}

\section{INTRODUCCION}

Uno de los más molestos síntomas de la edad avanzada y de la paridad en la mujer es la insidiosa y progresiva pérdida del control urinario. Si bien el problema generalmente comienza con la infrecuente pérdida de la continencia de la orina en casos donde hay un súbito aumento de presión sobre el diafragma pelviano, el problema de la incontinencia urinaria aumenta gradualmente con la edad hasta el grado de que la paciente debe usar un apósito en todo momento para evitar

* Jefe de la Sección de Ginecología y Posgrado, Facultad de Medicina, Universidad. Nacional.

Jefe Sección de Ginecología de la Clínica de Fray Bartolomé de las Casas, Caja de Previsión Social de Bogotá.

** Residente III, Depto. de Ginecología. y Obstetricia, Facultad de Medicina, Universidad Nacional. mojar la ropa. En este momento se vuelve difícil para la paciente continuar negando que este síntoma ha complicado su vida personal, familiar y social. Este síntoma puede asociarse con el uretrocele y el cistocele, causado por un relajamiento de la porción anterior del diagrama pelviano y urogenital. La hernia de la uretra y de la base de la vejiga a través del diagrama pelviano, suele ocurrir concomitantemente como resultado del estiramiento y relajación del sostén aponeurótico de los músculos del piso pelviano, durante el paso del feto por el conducto vaginal.

La debilidad de la musculatura del diafragma pélvico puede ocurrir como exclusivo resultado de la edad, con la atrofia muscular y relajación similar del diafragma urogenital subyacente. En las nulíparas, la relajación del sostén muscular puede suceder en la edad avanzada con el singular desarrollo de un cistocele sin uretrocele. En éste caso el molesto síntoma de la presión pelviana, como resultado 
de un cistocele progresivamente en aumento se produce con el acompañamiento de incontinencia urinaria de esfuerzo.

Las pacientes afectadas sufren una serie de molestias que van desde la interrupción de la actividad social en el medio que se desenvuelven hasta los trastornos producidos por los fenómenos de irritación local por la orina que las lleva a buscar consejo médico (1).

En 1913, Kelly de John's Hopkins Hospital informó de una técnica específica de reparación y aplicación de la pared anterior de la vagina, la llamada operación de fruncimiento de Kelly, la cual fue precursora de la vía de acceso vaginal para la corrección operatoria de la Incontinencia Urinaria de Esfuerzo. Casi simultáneamente, tres ilustres Ginecólogos Europeos, Gobbel en 1910, Frangenheim en 1914 y Stoeckel en 1917, preconizaron el uso de operaciones suprapúbicas utilizando cabestrillos musculares o aponeuróticos para reparar el defecto ana tómico considerado, causa de la fuga normal de orina.

En los años sucesivos se han logrado notables progresos en nuestros conocimientos sobre los trastornos fisiológicos y anatómicos subyacentes que causan éste síntoma tan molesto.

A partir de 1949 se comenzaron a describir una serie de técnicas quirúrgicas para la corrección de la Incontinencia Urinaria de Esfuerzo, cuyo objetivo fundamental es la elevación del cuello vesical o tejidos parauretrales a estructuras posteriores del pubis, de la índole del cartílago de la sinfisis púbica, del periostio de la rama del pubis, vaina del recto o ligamento de Cooper entre las cuales están las técnicas de Marshall,
Marchetti y Krantz en 1949, Pereyra en 1959, Burch en 1961, Tanagho en 1976, Stamey en 1980, que han dado una efectividad de 90 a $93 \%(3,4,5,6,7)$.

Por otra parte, los conocimientos más precisos de los diferentes tipos de anatomía anormal involucrada han permitido comprobar que la vía vaginal no logra a menudo cura satisfactoria a largo plazo.

Hodgkinson (1970) (10), analizó el sitio que ocupa la colporrafia anterior en la cirugía para Incontinencia. Se ha informado cifras de curación entre 48 y $60 \%$. Jeficoste (1966), 60\%. Ross y Shingleton (1919), 57\%. Low (1967), $48 \%$. Gómez y Lomanto (1980) $48 \%$ (11). Sin embar. go, Green informa que la cifra excede de $90 \%$ (1975), por lo tanto se concluye que la vía vaginal es de poca efectividad en el manejo de la Incontinencia Urinaria de Esfuerzo. La vía suprapúbica en las múltiples publicaciones sobre el tema resulta ser muy superior para el manejo de la Incontinencia Urinaria de Esfuerzo observándose una eficacia del 85 al 95\% $(12,13)$.

En caso de Incontinencia Urinaria de Esfuerzo con Celes, se ha intentado mejorar los resultados obtenidos combinando en la misma paciente las técnicas abdominales y vaginales, en dos tiempos quirúrgicos, aumentando el éxito pero aumentando la morbilidad y la estancia hospitalaria $(13,14)$.

Por lo tanto, por todo lo anterior, nos ha motivado proponer el análisis de la suspensión endoscópica del cuello vesical en casos de Incontinencia Urinaria de Esfuerzo acompañada de Celes.

En 1973, Stamey describe una operación que tiene las siguientes ventajas: 
a. Una correcta identificación del cuello vesical al tiempo de la cirugía (7).

b. Soporta fuertemente el cuello vesical mediante la unión de la fascia pubocervical con sutura no absorbible polipropileno pasada a través de un tubo de dacrón.

c. No requiere cirugía pélvica abierta y por lo tanto disminuye la morbilidad operatoria.

d. Permite mediciones fisiológicas durante la cirugía que aseguran al cirujano que la incontinencia ha sido corregida.

En 1980, Stamey $(15,8,9)$, publica una serie de 203 casos con un éxito del $91 \%$. Utiliza la técnica de Pereyra, según como se describió anteriormente, pero además utiliza agujas para levantar las estructuras paracervicales, complementa la técnica con el empleo de procedimientos endoscópicos intraoperatorios y en esta forma, controlar de manera adecuada la ubicación del cuello vesical y a la vez comprobar la integridad vesical y además deja catéter suprapúbico para el drenaje vesical postoperatorio, notando disminución en la estancia hospitalaria y la infección de vías urinarias.

\section{OBJETIVOS}

Nos proponemos:

1. Conocer la eficacia de la suspensión endoscópica del cuello vesical en el tratamiento de la Incontinencia Urinaria de Esfuerzo en la mujer, acompañada de Celes.

2. Conocer los riesgos intraoperatorios y postoperatorios de ésta técnica quirúrgica en nuestra consulta de incontinencia.
3. Disminuir la estancia hospitalaria de nuestros pacientes.

\section{MATERIAL Y METODOS}

Se realizó un estudio prospectivo de tipo descriptivo donde se evaluó la suspensión endoscópica del cuello vesical en el manejo de la Incontinencia Urinaria de Esfuerzo en la mujer acompañada de celes y se presenta un informe preliminar de 50 pacientes tratadas en la consulta de Ginecología en el Hospital Materno Infantil y la Clínica Fray Bartolomé de las Casas de Bogotá desde julio de 1984 a septiembre de 1985. Se seleccionaron las pacientes con incontinencia urinaria de esfuerzo Grado II y III acompañada de celes.

Se incluyeron tres pacientes que no presentaban celes.

Las pacientes se manejaron de la siguiente manera: (Ver Gráficas 1 y 2 ).

\section{DESCRIPCION DE LA TECNICA OPERATORIA}

A todas las pacientes se les practicó la SUSPENSION ENDOSCOPICA DEL CUELLO VESICAL MAS CORRECCION DE LOS CELES en el mismo acto quirúrgico y cuya descripción de la técnica es la siguiente:

La paciente es colocada en posición de litotomía modificada. Después del lavado y de la antisepsia del campo operatorio se coloca una sonda de Foley No. 16.

\section{Incisión Abdominal}

Se hacen dos incisiones suprapúbicas de 2 a $3 \mathrm{cms}$ de largo a la izquierda y a la 
GRAFICA No. 1

CONSULTA EXTERNA DE GINECOLOGIA<smiles>[Y]C</smiles>

DIAGNOSTICO DE LA INCONTINENCIA URINARIA DE ESFUERZO
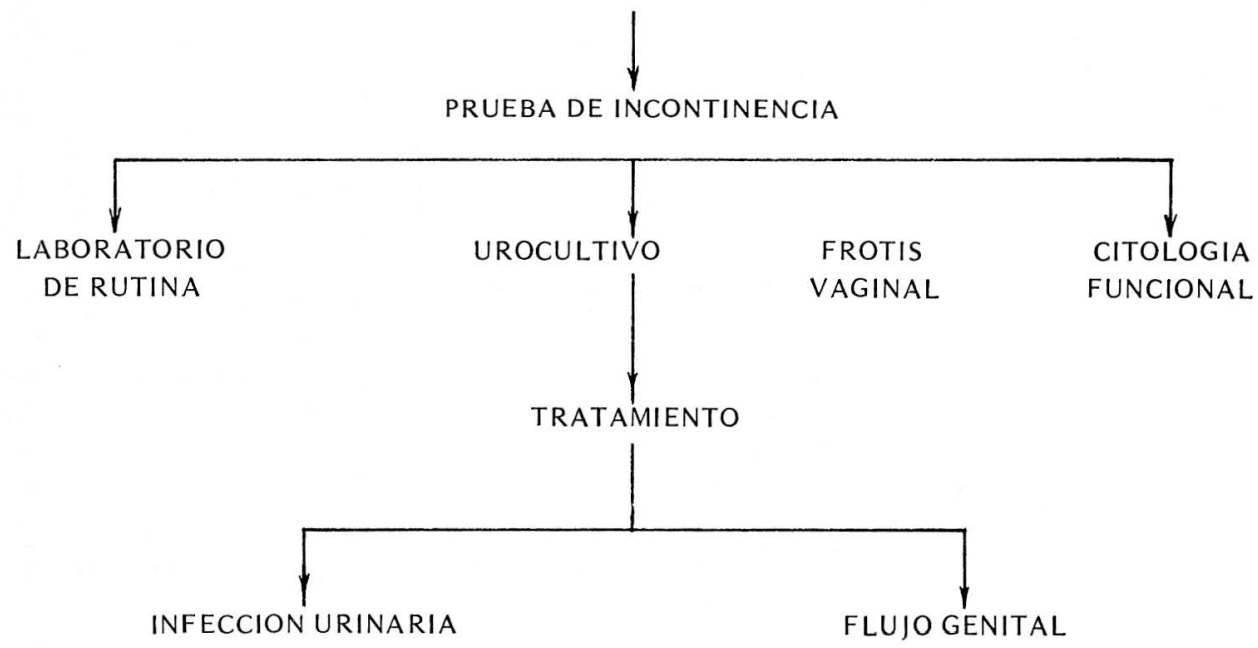

derecha de la línea media, exactamente en el borde superior de la sínfisis púbica y profundamente hasta la fascia de los rectos.

\section{Incisión Vaginal}

Si la uretra es corta ( $4 \mathrm{cms}$ o menos) la incisión transversal se inicia justamente por debajo de meato urinario. Si la uretra es larga la incisión puede hacerse cerca del cuello vesical. Cualquier liberación de la pared anterior de la vagina en la región del cuello vesical debe ser cuidadosa para evitar debilitamiento de la fascia pubocervical.

\section{Agujas de Stamey}

Se han descrito tres agujas especiales muy simples: UNA RECTA, otra con
ANGULO DE 15 GRADOS y otra con ANGULO DE 30 GRADOS. Se prefiere siempre comenzar con la aguja recta a partir del borde interno de cualquiera de las incisiones abdominales. Las agujas anguladas son especialmente útiles en pacientes con cirugía previa. Se introduce la aguja a través de la fascia del recto anterior y se guía hacia abajo exactamente al lado del cuello vesical (indicado por el balón de la sonda) y hacia la incisión vaginal. Es importante enfatizar que la única parte de la cirugía en que el avance es ciego, es el primer centímetro de introducción manual, el resto es siempre bimanual dirigido.

\section{Uso de la Endoscopia}

Con el uretrocistoscopio se revisa que la aguja no haya penetrado en la vejiga, ni 


\section{GRAFICA No. 2}

CONSULTA DE LA CLINICA DE INCONTINENCIA<smiles>[Y][V]</smiles>

INCONTINENCIA URINARIA DE ESFUERZO GRADO II Y III<smiles>[Y]C</smiles>

ELABORACION DE LA HISTORIA CLINICA



En los casos en los cuales existen manifestaciones clínicas en donde es necesario descartar patología funcional

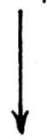

INCONTINENCIA URINARIA DE ESFUERZO DE MANEJO QUIRURGICO

INCONTINENCIA URINARIA DE ESFUERZO ACOMPAÑADA DE CELES<smiles>FC(F)(F)[Te]</smiles><smiles>[Y]C</smiles>

INTERVENCION QUIRURGICA CON LA TECNICA DE SUSPENSION ENDOSCOPICA DE CUÉLLO VESICAL MAS COLPORRAFIA ANTERIOR COLPORRAFIA POSTERIOR Y PERINEORRAFIA



MANEJO DE LAS COMPLICACIONES 
se haya ubicado en la submucosa o en la capa muscular. Luego se revisa el cuello vesical y se observa si la aguja está bien ubicada por los movimientos de los tejidos adyacentes fácilmente detectables. Es la parte más importante de la operación y es la razón por la cual se denomina suspensión endoscópica del cuello vesical.

Se introduce la sonda de Foley, para luego introducir de nuevo la aguja de Stamey a $1 \mathrm{~cm}$ por fuera de la anterior procediendo de la misma manera que para la primera aguja. Tanto en la porción vaginal como en la porción suprapúbica del Polipropileno se coloca un tubito de teflón de $1 \mathrm{~cm}$ de largo por $5 \mathrm{~mm}$ de ancho para evitar que el material de sutura corte las estructuras. Luego se enhebra el polipropileno en el ojo de la aguja y se retira hacia arriba y se traccionan hacia arriba los dos cabos. Igual procedimiento se practica en el otro lado. Localizándose entonces dos suturas de suspensión a cada lado del cuello vesical.

Una vez establecidas las asas suspensorias a cada lado del cuello vesical se introduce el uretrocistoscopio para observar el punto de cierre uretral cuando cada asa suspensoria es traccionada.

Con el uretrocistoscopio en la uretra distal, el Cirujano puede determinar:

- Que el cierre ocurre exactamente en el cuello vesical.

- Que las asas suspensorias estén ubicadas simétricamente.

- La cantidad de tensión requerida para cerrar el cuello vesical, lo que le da una idea de qué tanto se deben apretar los nudos posteriormente.
La incisión vaginal se cierra primero con catgut cromado $2-0$ antes de anudar las suturas de polipropileno suprapúbico. Las suturas suprapúbicas se suturan a la menor tensión posible.

Finalmente, se introduce una sonda Foley, se llena la vejiga a capacidad y se pasa un catéter suprapúbico percutáneo No. 10 F. a 3 cms. por encima de la sínfisis púbica. Posteriormente se retira la sonda de Foley y la paciente es llevada a la sala de recuperación.

PROCESAMIENTO Y PRESENTACION DE RESULTADOS

El análisis de los resultados se presentan con la ayuda de Tablas y Gráficas.

\section{LA CERVICOSUSPENSION}

ENDOSCOPICA EN EL TRATAMIENTO

DE LA INCONTINENCIA URINARIA DE ESFUERZO (I.U.E.)

FIGURA No. 1

EDAD

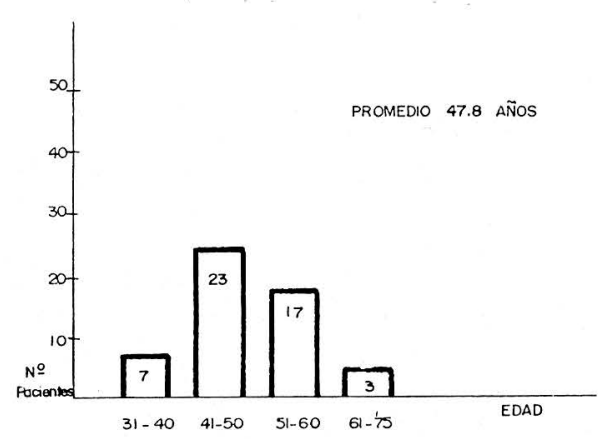


FIGURA No. 2

PARIDAD

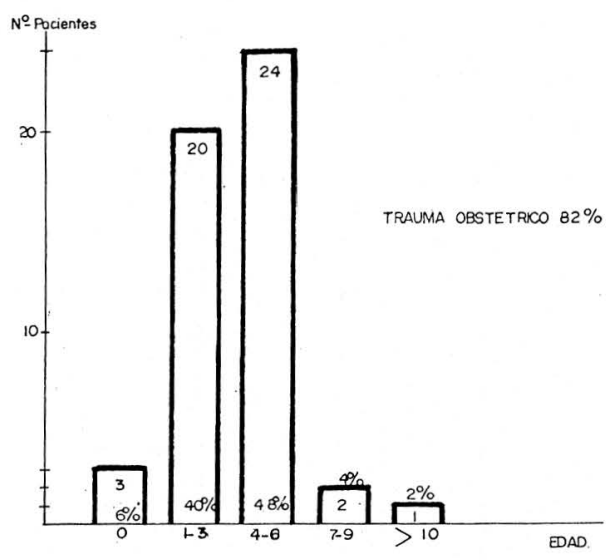

FIGURA No. 3

\section{EVOLUCION}

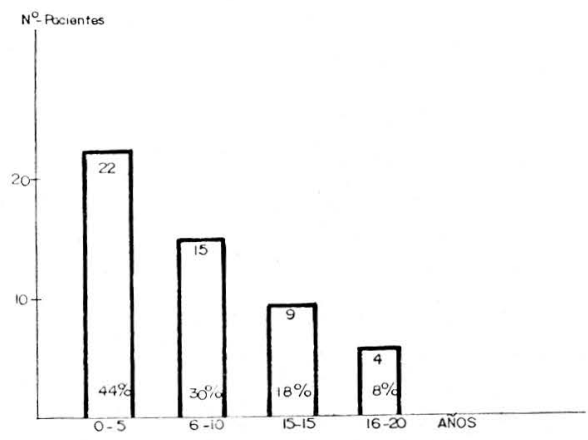

FIGURA No. 4 GRADO DE INCONTINENCIA
URINARIA DE ESFUERZO II 20-40 $\square$ III 30.60 圈

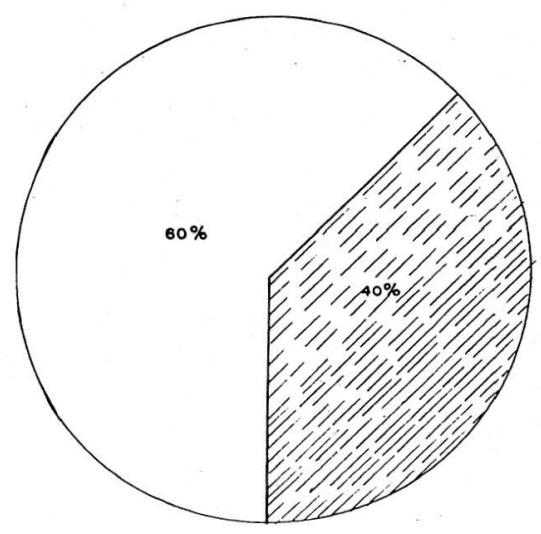

FIGURA No. 5

GRADOS DE URETROCISTOCELES

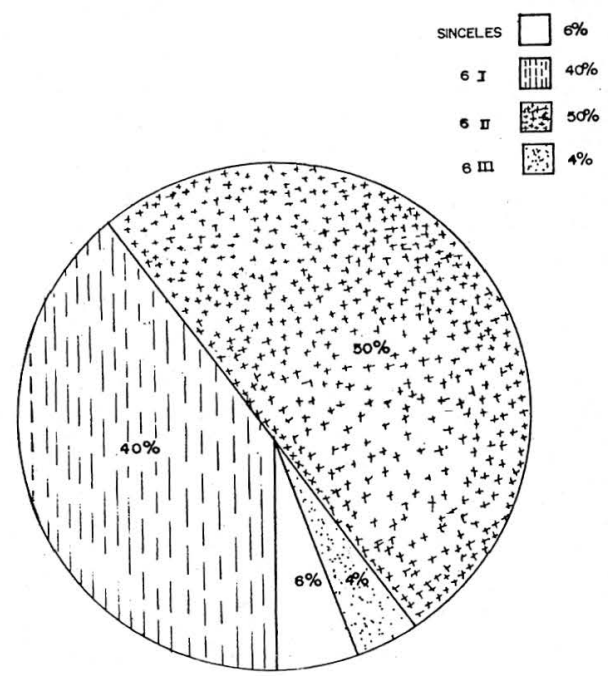


FIGURA No. 6

GRADO DE RECTOCELE

Y DESGARRO PERINEAL

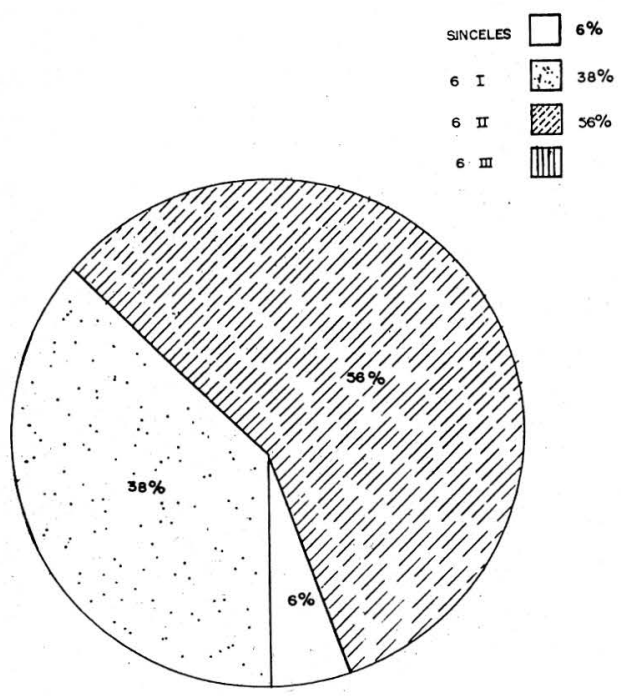

FIGURA No. 7

\section{PROCEDIMIENTO QUIRURGICO}

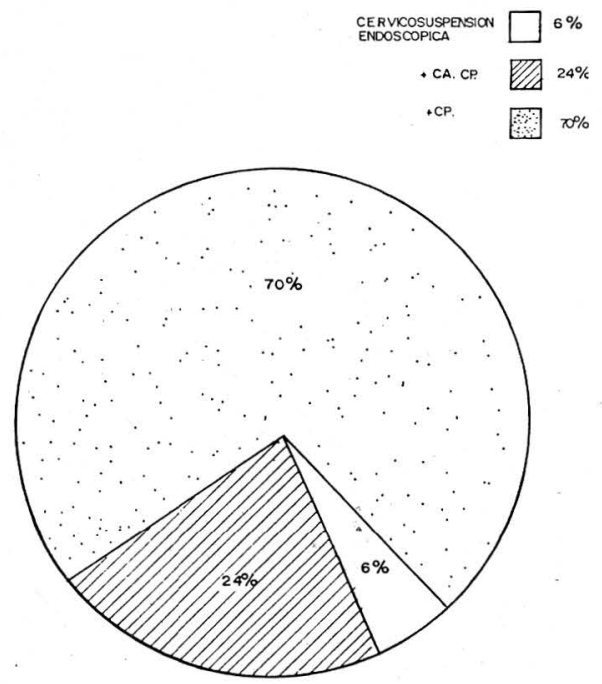

FIGURA No. 8

\section{PROCEDIMIENTOS \\ PARACLINICOS}

FIG: 7. PROCEDIMIENTOS PARACLINICOS

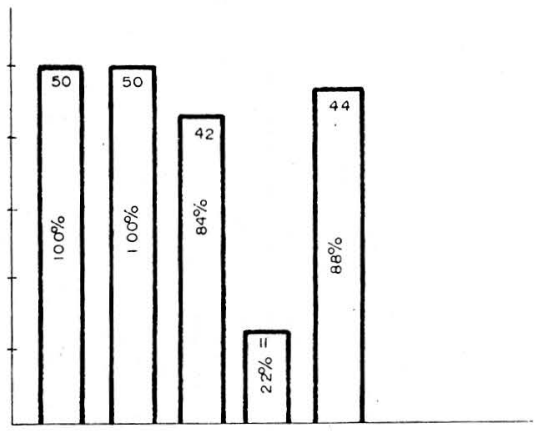

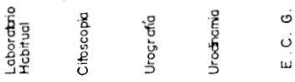

TIEMPO QUIRURGICO

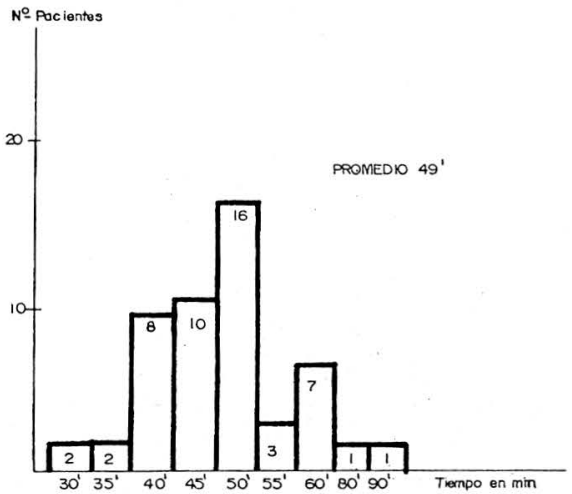


FIGURA No. 10

COMPLICACIONES

POSTOPERATORIAS
FIGURA No. 12

NUMERO DE DIAS

DEL CATETER SUPRAPUBICO

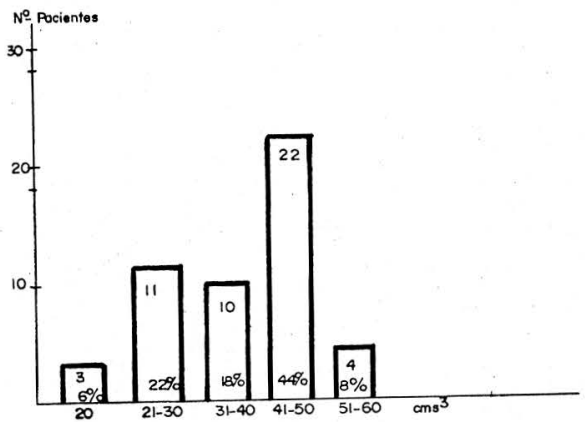

FIGURA No. 13

SEGUIMIENTO

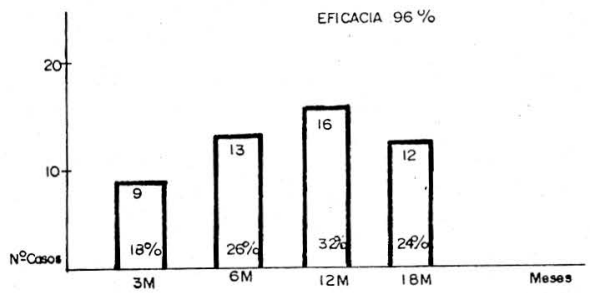

FIGURA No. 14

ESTANCIA HOSPITALARIA

\section{RESIDUO VESICAL}
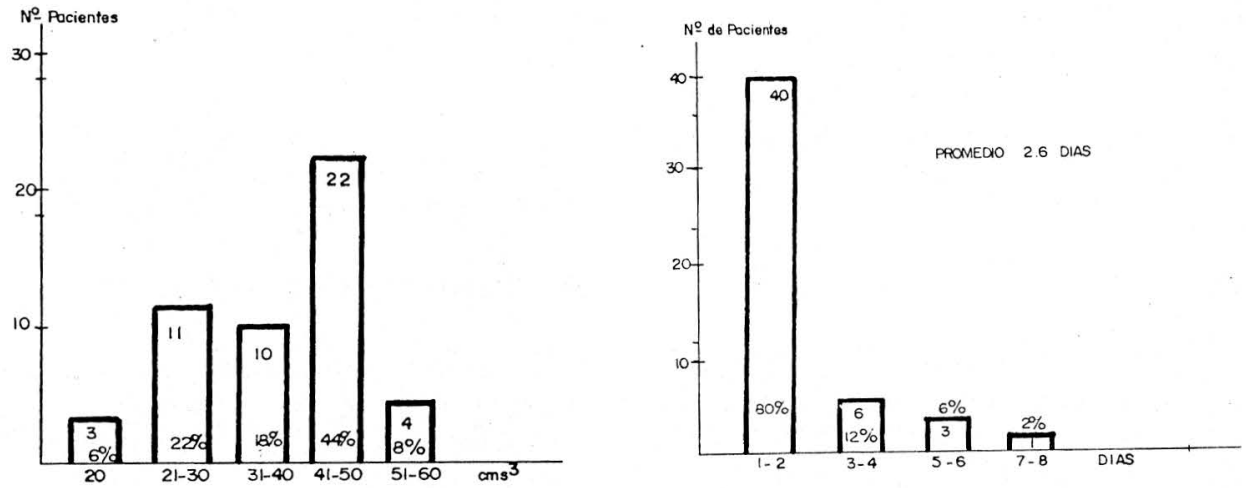


\section{RESULTADOS}

\section{INFORMACION PREOPERATORIA}

Las pacientes a quienes se les practicó la suspensión endoscópica del cuello vesical para la corrección de su incontinencia urinaria de esfuerzo tenían las siguientes características:

EDAD: la menor de las pacientes tenía 35 años y la mayor de ellas 73. El 23 (46\%) de las pacientes estuvo entre los 41 y 50 años y el promedio de edad fue de 47.8 años, (Figura No. 1).

PARIDAD: 47 (94\%) pacientes eran multíparas de las cuales el $82 \%$ tenían antecedentes de trauma obstétrico. Hubo tres casos de multíparas y un caso de paridad de 12 (Figura No. 2).

EVOLUCION: El mínimo tiempo de evolución fue de un año y el máximo de 20 años. $22(44 \%)$ pacien'tes tenían una evolución menor de 5 años y $4(8 \%)$ entre los 16 y 20 años (Figura No. 3).

OBESIDAD: $24(48 \%)$ pacientes eran obesas.

INTERVENCIONES PREVIAS: 8 pacientes $(16 \%)$ tenían antecedentes de cirugía de Kelly y una $(2 \%)$ tenían antecedente de cirugía de suspensión endoscópica del cuello vesical para corrección de incontinencia urinaria de esfuerzo. Además $6(12 \%)$ tenían antecedentes de Histerectomía (Tabla No. 1).

\section{DIAGNOSTICO CLINICO}

TIPO DE INCONTINENCIA: 20 (40\%) pacientes tenían una incontinencia urinaria de esfuerzo Grado II y $30(60 \%)$ tenían IUE Grado III (Figura No.4).

\section{CLASIFICACION DE LOS CELES}

URETROCISTOCELES: 20 (40\%) pacientes tenían UCC Grado I, 25 (50\%) Grado II y 2 (4\%) Grado III. $3(6 \%)$ pacientes no presentaron celes (Figura No. 5).

RECTOCELES Y DESGARRO PERINEAL: 19 pacientes (38\%) tenían rectoceles y desgarro perineal Grado I, 26 (52\%) Grado II, $3(6 \%)$ no presentaron celes (Figura No. 6).

\section{PRUEBAS DE INCONTINENCIA}

$25(50 \%)$ pacientes tenían pruebas de incontinencia positiva de pie, 19 (38\%) pacientes tenían prueba de incontinencia positiva de decúbito $y$ todas las pacientes presentaron prueba de Bonney favorable.

\section{METODOS PARACLINICOS}

A la totalidad de las pacientes se les practicaron los exámenes paraclínicos preoperatorios habituales incluyendo los urocultivos, los cuales fueron negativos. A todas las pacientes se les practicó citoscopia descartando anormalidades uretrovesicales.

A $42(84 \%)$ pacientes se les practicó urografía descartando anormalidades del trac to urinario alto.

A $11(22 \%)$ pacientes se les practicó Urodinamia por existir sintomatología sugestiva de patología funcional.

\section{PROCEDIMIENTO QUIRURGICO}

De acuerdo a los grados de celes que acompañaban la incontinencia urinaria de esfuerzo se practicaron los siguientes procedimientos: 
A $35(70 \%)$ pacientes se les practicó suspensión endoscópica del cuello vesical más colporrafia posterior.

A $12(24 \%)$ pacientes se les practicó suspensión endoscópica del cuello vesical más colporrafia anterior y posterior (Figura No. 7).

A $3(6 \%)$ pacientes se les practicó suspensión endoscópica del cuello vesical que correspondió a las pacientes nulíparas.

TIEMPO QUIRURGICO: El tiempo promedio fue de 49.1 minutos y osciló entre 30 minutos y 90 minutos.

En la suspensión endoscópica del cueIlo vesical sola el tiempo promedio fue de 31.6 minutos.

En la suspensión endoscópica del cueIlo vesical más colpoperineorrafia posterior el tiempo promedio fue de $53.7 \mathrm{mi}$ nutos.

$Y$ en la suspensión endoscópica del cuello vesical más colporrafia anterior y posterior el tiempo promedio fue de 55.0 minutos (Figura No. 8).

\section{COMPLICACIONES OPERATORIAS:}

Entre las complicaciones intraoperatorias solamente hubo $3(6 \%)$ casos de perforación vesical las que fueron diagnosticadas bajo la visión endoscópica, habiendo sido necesario pasar nuevamente las agujas por el trayecto descrito.

CATETER SUPRAPUBICO: Se utiliza en esta cirugía drenaje suprapúbico con catéter No. 10 en todas las pacientes sin complicaciones y el tiempo promedio de permanencia del catéter fue de 3.28 días, a $34(68 \%)$ pacientes se les retiró a las 48 horas. En 6 (12\%) pacientes. la permanencia fue de 8 días.

\section{MEDICACION POSTOPERATORIA:} No se utilizaron antibióticos profilácticos ni antisépticos urinarios.

COMPLICACIONES POSTOPERATORIAS: Una (2\%) paciente presentó infección en la herida quirúrgica suprapúbica; otra $(2 \%)$ presentó infección en la colporrafia. $3(6 \%)$ pacientes presentaron granulomas. No se presentaron fístulas. $27(54 \%)$ pacientes presentaron dolor leve, $19(38 \%)$ presentaron dolor moderado y una $(2 \%)$ dolor severo.

INFECCION URINARIA: $8(16 \%)$ pacientes presentaron infección urinaria comprobada con urocultivo; 7 (14\%) presentaron urocultivo positivo a las 48 horas de la cirugía y una $(2 \%)$ presentó urocultivo positivo a los 8 días.

El germen más común fue el $\mathrm{E}$. Coli $(62.5 \%)$. Otros gérmenes fueron el Estafilococo Epididimos $17.5 \%$, el Enterobacter $15 \%$, y el Proteus $5 \%$.

El tratamiento estuvo de acuerdo al antibiograma.

RETENCION URINARIA: 2 pacientes (4\%) presentaron retención urinaria por 8 días, por lo cual se procedió a retirar un punto de la sutura de la suspensión endoscópica con lo cual obtuvieron la micción espontánea. Estas dos pacientes permanecen continentes.

ESTANCIA: de 40 pacientes tuvieron un promedio de 4 días, $3(6 \%)$ tuvieron un promedio de 6 días y una $(2 \%)$ paciente tuvo un promedio de 8 días. El promedio de estancia hospitalaria fue de 2.6 días.

SEGUIMIENTO: 10 pacientes Ilevan 18 meses de seguimiento, $16(32 \%)$ pacientes llevan un año y $24(48 \%)$ pacientes llevan seis meses. 
EFICACIA: Con el fin de evaluar la continencia establecida por esta técnica quirúrgica no solamente se tuvieron en cuenta las manifestaciones subjetivas de la paciente sino que fueron tenidas como continentes aquellas que con cantidades de un promedio de $250 \mathrm{CC}$ de orina en vejiga y con aumentos de la presión intraabdominal (tos, pujo, estornudo) no se evidencia salida de orina.

Basados en los anteriores parámetros se pudo comprobar que $48(96 \%)$ pacientes tenían continencia urinaria de esfuerzo a los 6 meses.

Es de anotar que las 10 pacientes seguidas a 18 meses están continentes $y$ las 16 seguidas a un año también están continentes.

FRACASOS: Se presentaron dos fracasos; en una paciente antes de los tres meses y en otra antes de los 6 meses. Una de las cuales se reintervino con la misma técnica y hasta el momento está continente.

\section{COMENTARIOS}

EDAD Y PARIDAD: De acuerdo con los resultados obtenidos corroboran la información ya conocida del hecho de que la incontinencia urinaria de esfuerzo es más frecuente en la perimenopausia, íntimamente relacionada con la multiparidad y el trauma obstétrico.

DIAGNOSTICO: La técnica de la suspensión endoscópica del cuello vesical se practicó en pacientes con incontinencia urinaria de esfuerzo tipo II y tipo III y el 94\% tenían celes de I a III; además presentaron prueba de Bonney favorable. El $18 \%$ de las pacientes tenían antecedentes de cirugía para corrección de su incontinencia urinaria de esfuerzo. $Y$ el $50 \%$ eran pacientes obesas.
PROCEDIMIENTO QUIRURGICO: $\mathrm{el}$ $74,5 \%$ de las pacientes el uretrocistocele corrigió con la suspensión endoscópica del cuello vesical lo cual favoreció el tiempo quirúrgico. Las variaciones en el tiempo quirúrgico estuvieron supeditadas a que en unas pacientes el uretrocistocele se corrigió con la suspensión endoscópica del cuello vesiçal.

COMPLICACIONES POSTOPERATO. RIAS: El accidente intraoperatorio no. ocasionó complicaciones en el seguimiento de las pacientes.

\section{LA INFECCION DE LA HERIDA} QUIRURGICA y de las colporrafias fue mínima. El dolor es un inconveniente de la técnica. No se presentaron fístulas. Se redujo la infección urinaria la cual se manifestó en el $16 \%$ de las pacientes y estuvo relacionada con el uso del catéter sup rapúbico.

La retención urinaria fue reducida al $4 \%$, y a pesar de que su tratamiento fue retirar un punto de la sutura de la suspensión endoscópica, las pacientes continuan continentes.

EFICACIA: La eficacia de la suspensión endoscópica es de $96 \%$ pero se debe esperar hacer el seguimiento a dos años para ratificar la verdadera eficacia.

ESTANCIA: Se observa una reducción de la estancia hospitalaria con esta técnica quirúrgica a un promedio de 3 días que estuvo supeditada a la menor morbilidad.

\section{CONCLUSIONES}

Consideramos la suspensión endoscópica del cuello vesical para la corrección de la incontinencia urinaria de esfuerzo, 
una técnica cuyas características la sitúan en un plano de franca superioridad sobre los procedimientos tanto de tipo suprapúbico como los empleados por la vía vaginal por los siguientes hallazgos obtenidos en el presente estudio:

1. - La alta incidencia de obesidad (48으) no fue obstáculo importante en el acto quirúrgico y por lo tanto no repercutió como factor de morbilidad en el postoperatorio.

2. - El antecedente de cirugía previa para la corrección de la incontinencia urinaria de esfuerzo dificulta notablemente la reintervención; lo cual se obvia con esta técnica ya que no se compromete el espacio de Retzius, pues las agujas que se emplean no ocasionaron hematomas ni dificultaron la cirugía.

3. - Permite practicar la corrección de los celes asociados en el mismo acto quirúrgico.

4. - El tiempo quirúrgico disminuyó a la mitad en comparación a las técnicas suprapúbicas convencionales.

5. - La morbilidad intraoperatoria es mínima y con la salvedad que la corrección de la misma se puede hacer en el mismo acto quirúrgico.

6. - El empleo de catéter suprapúbico, no obstante ser método invasivo y controvertido es considerado de mucha utilidad para la facilidad de medir el residuo vesical, menor infección y la facilidad de manejo por parte de la paciente.

7. - Las complicaciones postoperatorias son menores. La infección de la herida quirúrgica y de las colporrafias es mínima y se redujo la frecuencia de infección urinaria y de retención urinaria.
8. - Siendo la única técnica capaz de comprobar la ubicación del cuello vesical para hacerlo intraabdominal, tiene el menor porcentaje de retención urinaria postoperatoria.

9. - La estancia hospitalaria es desproporcionalmente menor con relación a las técnicas similares, fruto de la baja morbilidad.

10. - Por el hecho de suspender la fascia endopélvica sobre la aponeurosis de los rectos-es relativamente frecuente el dolor moderado a severo.

11. - Se reconoce la necesidad de hacer un seguimiento mayor de las pacientes para concluir en forma definitiva la verdadera eficacia de la técnica.

12. - Sin lugar a dudas es una técnica que exige completo dominio por parte del cirujano de la endoscópica urológica, lo que se traduce como un obstáculo para la utilización en Ginecología.

13. - Es importante anotar que en este estudio prospectivo no se presentaron fístulas.

14. - Como desventajas relativas del procedimiento es importante anotar la posibilidad de lesiones tanto uretrales comio vesicales pero que se. pueden corregir en el acto quirúrgico.

15. - Es la técnica posiblemente ideal para aquellas pacientes que por diferentes razones ha sido comprometida la pared abdominal como aquellos casos de intervenciones ginecológicas de tipo sépticos y que han sido irradiadas previamente.

16. - En consideración a la baja morbilidad seria indicada en las pacientes con al to riesgo quirúrgico. 
17. - La eficacia es aceptable y los dos fracasos estuvieron relacionados con la técnica quirúrgica.

18. - Para poder llegar a un diagnóstico exacto de la incontinencia urinaria de esfuerzo, clave para el éxito operatorio final, es necesario la realización de una historia clínica y un examen físico completo.

19. - Se comprobó, como la mayoría de los autores, que la incontinencia urinaria es más frecuente en los grados menores de uretrocistoceles, en las grandes multíparas y entre los 40 y 50 años (edad promedio 47.8 años).

\section{RESUMEN}

Se analizan los resultados de 50 casos del primer estudio prospectivo realizado en el país para el tratamiento de la Incontinencia Urinaria de Esfuerzo (IUE) en la mujer, mediante la Suspensión Endoscópica del Cuello Vesical (SECV) y cistostomía, desde abril de 1984 hasta septiembre de 1985 .

El $40 \%$ presentaba IUE tipo II y el $60 \%$ tipo III. El 94\% tenía celes conco-

\section{BIBLIOGRAFIA}

1. TE LINDE B. W., Incontinencia de Orina al Esfuerzo Uretrocele y cistocele. Ginecología Operatoria: 456 . 472; 1980 5a. Edición.

2. KELLY H. A., Incontinence of Urine in Women Urologic and Cutaneous Review 17. 291-293, 1913.

3. MARSHALL V. F., MARCHETTI A. A., KRANTEZ K. E., The correction of mitante y el $30 \%$ antecedentes de cirugías ginecológicas. Se observó obesidad en el $48 \%$ En el $97 \%$ de los casos se practicó SECV con corrección de celes y el promedio de tiempo quirúrgico fue de 49 minutos. Como complicación operatoria se anota la perforación vesical en el $6 \%$ de los casos, y en el postoperatorio granulomas de pared (6\%), abscesos (2응 e infección de la colpoperineorrafia (2\%). El drenaje por cistostomía duró en promedio 3.3. días, observándose infección urinaria en el $16 \%$ de los casos y retención urinaria posterior en el $4 \%$ Veintiseis (26) pacientes tuvieron seguimiento de 12 a 18 meses, con resultados exitosos en todas. Se presentaron dos fracasos antes de los seis meses, imputables a fallas en la técnica quirúrgica.

Con los resultados obtenidos los autores consideran provisionalmente la SECV como la técnica ideal para el manejo de la IUE asociada con celes, obesidad y antecedentes de cirugías ginecológicas, en razón a la ubicación adecuada del cuello vesical, al menor tiempo quirúrgico y a la baja morbilidad. Se recomienda sistematizar el drenaje mediante cistostomía. Un seguimiento mayor de las pacientes permitirá obtener conclusiones definitivas.
Stress Incontinence by Simple-Vesico Urethral. Campell Urology-Vol 3 Philadelphia Saunders. 2292, 1980.

4. PEREYRA A. J. LEBERTZ T. B., Combined Uretrovesical Suspension and Vagino-Urethroplasty for Correction of Urinary Stress Incontinence. Obstetrics and Gynecology. 30, 537-546, 1967.

5. BURCH J. C., Cooper Ligament Urethrovesical for Stress Incontinence. Am J. Of Obstetrics Gynecology 100, 764, 772, 1968. 
6. TANAGHO E. A., Colpocystourethropexy. The Way We do it, J. Urology. 116: $751-753,1976$.

7. STAMEY T. A., Endoscopic Suspension of Vesical Neck for Urinary Incontinente. Surg. Gynecol Obstet. 136, 547, 554, 1973.

8. STAMEY T. A., Urinary Incontinence in the Female. Camphell Urology (3): 2272-2293. 1979.

9. STAMEY T. A., Endoscopic Suspension of the Vesical Neck for Urinary Incontinence in Females. Report on 203 consecutive patients Annals of Surgery. 192 (4) 465-471, 1980.

10. HODKINSON C. P., Stress Urinary Incontinence. Am J. Obstet. Gynecol. 108, 1141, 1970.

11. GOMEZ P. Jr., LOMANTO A., Operación de Kelly. Rev. Colombiana de Obstetricia y Ginecología. Vol. 34 No. 1, 28-34, 1983.
12. LOMANTO A., GOMEZ P. Jr., Evaluación de la Colpocistouretropexia en la Incontinencia Urinaria de Esfuerzo. Revista Colombiana de Obstetricia y Ginecología. Vol. XXXIII, pag. 112, 1982.

13. POWELL L. C. Jr., Retropubic Urethrocystopexy: Vaginal Approach. Am. J. Obstet. Gynecol. 140: 91, 1981.

14. LOPEZ A., CORZ̃O J., RODRIGUEZ H., Corrección de la Incontinencia Urinaria de Esfuerzo por medio de la Uretrocistopoxia Retropúbica por vía vaginal. Revista Colombiana de Obstetricia y Ginecología. Vol. XXXIV No. 1, 1983.

15. STAMEY T. A., SCHAEFFER A. J., CONDOY M., Clinical and Roentgenographic Evaluation of Endoscopic Suspension of the Vesical Neck for Urinary Incontinence. Surg. Gynecol Obstet. 140: 355-360, 1975. 